\title{
A NOTE ON MULTIVALUED DIFFERENTIAL EQUATIONS ON PROXIMATE RETRACTS
}

\author{
DONAL O'REGAN \\ National University of Ireland \\ Department of Mathematics \\ Galway, Ireland
}

(Received October, 1997; Revised July, 1998)

This paper discusses viable solutions for differential inclusions in Banach spaces. Existence will be established in two steps. In step 1, a nonlinear alternative of Leray-Schauder type [8] for maps with closed graphs will be used to establish a variety of existence principles for the Cauchy differential inclusion. Step 2 involves using the results in step 1 together with some tricks involving the Bouligand cone (and sometimes the Urysohn function) so that new existence criteria can be established for multivalued differential equations on proximate retracts.

Key words: Viable Solutions, Multivalued, Differential Inclusions, Proximate Retracts.

AMS subject classifications: $34 \mathrm{~A} 60$.

\section{Introduction}

In this paper we study the existence of solutions $y:[0, T] \rightarrow K \subseteq E$ (so called viable solutions) to the differential inclusion

$$
\left\{\begin{array}{l}
y^{\prime}(t) \in \phi(t, y(t)) \quad \text { a.e. } t \in[0, T] \\
y(0)=y_{0} \in K .
\end{array}\right.
$$

$K$ is a proximate retract and $\phi:[0, T] \times K \rightarrow 2^{E}$; here $E$ is a real Banach space and $2^{E}$ denotes the family of all nonempty subsets of $E$. Using a nonlinear alternative of Leray-Schauder type, we were able in [7] to establish some general existence principles and theory for (1.1) (however in [7] we had to assume $\phi$ was a $K$-Carathéodory map [4]). In this paper using some recent results of the author (see $[6,8])$ we are able to discuss a more general $\phi$.

The technique to establish the existence of viable solutions to (1.1) will be in two steps. In step one we discuss the differential inclusion 


$$
\left\{\begin{array}{l}
x^{\prime} \in F(t, x) \text { a.e. on }[0, T] \\
x(0)=x_{0} \in E
\end{array}\right.
$$

Our goal will be to establish some general existence principles for (1.2) which will automatically lead to new criteria for the existence of viable solutions to (1.1). We will discuss (1.2) in the introduction. The proofs of our existence principles will be elementary since all the analysis was completed in [6-8]. In Section 2 (which is step 2 ) we will first discuss directly (1.1) when $\phi$ is not necessarily $K$-Carathéodory. New results are presented which extend previously known results in the literature $[2,3,5$, 7. 9]. Then we will examine (1.1) indirectly; the idea in this case is to examine the differential inclusion

$$
\left\{\begin{array}{l}
x^{\prime} \in \overline{\exp } \overline{\operatorname{conv}} \phi(t, x) \quad \text { a.e. on }[0, T] \\
x(0)=y_{0} \in K
\end{array}\right.
$$

Remark 1.1: For a set $A, \exp (A)$ is the set of extreme points of $A$ and $\operatorname{conv}(A)$ is the convex hull of $A$.

Again new results will be obtained for (1.3) which will lead to new existence criteria for (1.1).

To conclude this section we discuss the differential inclusion (1.2) where $F:[0, T] \times E \rightarrow C(E)$ (here $C(E)$ denotes the family of all nonempty, compact subsets of $E)$. We look for solutions to $(1.2)$ in $W^{1,1}([0, T], E)$. Recall $W^{1,1}([0, T], E)$ is the set of continuous functions $u$ such that there exists $v \in L^{1}([0, T], E)$ with $u(t)-$ $u(0)=\int{ }_{0}^{t} v(s) d s$ for all $t \in[0, T]$. [Notice if $u \in W^{1,1}([0, T], E)$ then $u$ is differentiable almost everywhere on $[0, T], u^{\prime} \in L^{1}([0, T], E)$ and $u(t)-u(0)=\int_{0}^{t} u^{\prime}(s) d s$ for $t \in[0, T]$.$] Before we specify conditions on F$ we first recall some well known concepts [4]. Let $E_{1}$ and $E_{2}$ be two Banach spaces, $X$ a nonempty closed subset of $E_{1}$ and $S$ a measurable space (respectively $S=I \times E$, where $I$ is a real interval, and $A \subseteq S$ is $\mathcal{L} \otimes \Im$ measurable if $A$ belongs to the $\sigma$-algebra generated by all sets of the form $N \times D$ where $N$ is Lebesgue measurable and $D$ is Borel measurable). Let $H$ : $X \rightarrow E_{2}$ and $G: S \rightarrow E_{2}$ be two multifunctions with nonempty closed values. The function $G$ is measurable (respectively $\mathcal{L} \otimes \mathscr{B}$ measurable) if the set $\{t \in S: G(t) \cap B \neq \emptyset\}$ is measurable for any closed $B$ in $E_{2}$. The function $H$ is lower semicontinuous (l.s.c.) (respectively upper semicontinuous (u.s.c.)) if the set $\{x \in X: H(x) \cap B \neq \emptyset\}$ is open (respectively closed) for any open (respectively closed) set $B$ in $E_{2}$.

When we examine (1.2) we assume $F:[0, T] \times E \rightarrow C(E)$ satisfies some of the following conditions (to be specified later):

$$
\begin{gathered}
\left\{\begin{array}{l}
\text { (i) } t \mapsto F(t, x) \text { is measurable for every } x \in E \\
\text { (ii) } x \mapsto F(t, x) \text { is u.s.c. for a.e. } t \in[0, T]
\end{array}\right. \\
\left\{\begin{array}{l}
(i) t \mapsto F(t, x) \text { is measurable for every } x \in E \\
(i i) x \mapsto F(t, x) \text { is continuous for a.e. } t \in[0, T]
\end{array}\right. \\
\left\{\begin{array}{l}
\text { (i) }(t, x) \mapsto F(t, x) \text { is } \mathcal{L} \otimes \mathscr{B} \text { measurable } \\
\text { (ii) } x \mapsto F(t, x) \text { is l.s.c. for a.e. } t \in[0, T]
\end{array}\right.
\end{gathered}
$$


$\left\{\begin{array}{l}\text { for each } r>0 \text { there exists } h_{r} \in L^{1}[0, T] \text { such that }\|F(t, x)\| \leq h_{r}(t) \\ \text { for a.e. } t \in[0, T] \text { and every } x \in E \text { with }\|x\| \leq r\end{array}\right.$

$$
\left\{\begin{array}{l}
\text { there exists } h \in L^{1}[0, T] \text { such that }\|F(t, x)\| \leq h(t) \\
\text { for a.e. } t \in[0, T] \text { and all } x \in E
\end{array}\right.
$$

and

$$
\left\{\begin{array}{l}
\text { there exists } \gamma \geq 0 \text { with } 2 \gamma T<1 \text { and with } \\
\alpha(F([0, t] \times \Omega)) \leq \gamma \alpha(\Omega) \text { for any bounded subset } \Omega \text { of } E \\
\text { here } \alpha \text { denotes the Kuratowskii measure of noncompactness. }
\end{array}\right.
$$

We now present six existence principles for (1.2) which will be needed in Section 2.

Theorem 1.1: [8] Let $E=(E,\|\cdot\|)$ be a separable Banach space with $F:[0, T] \times$ $E \rightarrow C K(E)$ (here $C K(E)$ denotes the family of nonempty, compact, convex subsets of E). Assume (1.4), (1.7) and (1.9) hold. Also suppose there is a constant $M_{0}$, independent of $\lambda$, with $\|y\|_{0}=\sup _{t \in[0, T]}\|y(t)\| \neq M_{0}$ for any solution $y \in$ $W^{1,1}([0, T], E)$ to

$$
\left\{\begin{array}{c}
y^{\prime} \in \lambda F(t, y(t)) \quad \text { a.e. } t \in[0, T] \\
y(0)=x_{0}
\end{array}\right.
$$

for each $\lambda \in(0,1)$. Then $(1.2)$ has a solution $u \in W^{1,1}([0, T], E)$ with $\|u\|_{0} \leq M_{0}$.

Proof: Define the map $\mathscr{F}: C([0, T], E) \rightarrow 2^{L^{1}([0, T], E)}$ by

$$
\mathscr{F}(y)=\left\{v \in L^{1}([0, T], E): v(t) \in F(t, y(t)) \text { a.e. } t \in[0, T]\right\}
$$

and the map $S: L^{1}([0, T], E) \rightarrow C([0, T], E)$ by

$$
S v(t)=x_{0}+\int_{0}^{t} v(s) d s
$$

Solving $(1.10)_{\lambda}$ is equivalent to solving the fixed point problem

$$
y \in \lambda S \circ \mathscr{F}(y) \equiv \lambda N y
$$

here $N: C([0, T], E) \rightarrow 2^{C([0, T], E)}$. Let

$$
U=\left\{u \in C([0, T], E):\|u\|_{0}<M_{0}\right\}
$$

In Theorem 2.1 of [6] we showed

$$
N: C([0, T], E) \rightarrow C c(C([0, T], E))
$$

has closed graph and $N: \bar{U} \rightarrow C c(C([0, T], E))$ is condensing (here $C c(C([0, T], E))$ 
denotes the family of nonempty, closed convex subsets of $C([0, T], E))$. Now apply Theorem 3.1 of [8] to deduce the result.

Remark 1.2: Notice (1.9) can be replaced by any condition that guarantees that $N: \bar{U} \rightarrow C c(C([0, T], E))$ is condensing. Other types of conditions may be found in [3]; for example (1.9) may be replaced by

$$
\left\{\begin{array}{l}
\text { there exists } \gamma \geq 0 \text { with } 2 \gamma T<1 \text { and with } \\
\quad \lim _{h \rightarrow 0^{+}}+\alpha\left(F\left(J_{t, h} \times \Omega\right)\right) \leq \gamma \alpha(\Omega) \text { for } t \in(0, T] \\
\text { for any bounded subset } \Omega \text { of } E ; \text { here } \\
J_{t, h}=[t-h, t] \cap[0, T] .
\end{array}\right.
$$

A similar remark applies to Theorem 1.2, Theorem 1.3, Corollary 1.4, Theorem 1.5 and Corollary 1.6.

Theorem 1.2: [7] Let $E=(E,\|\cdot\|)$ be a separable Banach space and let $F$ : $[0, T] \times E \rightarrow C(E)$ satisfy (1.7), (1.9) and either (1.5) or (1.6). In addition, suppose there is a constant $M_{0}$, independent of $\lambda$, with $\|y\|_{0} \neq M_{0}$ for any solution $y \in$ $W^{1,1}([0, T], E)$ to $(1.10)_{\lambda}$ for each $\lambda \in(0,1)$. Then (1.2) has a solution $u \in$ $W^{1,1}([0, T], E)$ with $\|u\|_{0} \leq M_{0}$.

Proof: The proof follows from Theorem 2.2 of [7] (the only difference is that in this case $N_{1}$ (defined in [7]) is condensing).

Theorem 1.3: [6] Let $E$ be a separable Banach space and let $F:[0, T] \times E \rightarrow C K(E)$ satisfy (1.4), (1.7) and (1.9). Define the operator $N: C([0, T], E) \rightarrow 2^{C([0, T], E)}$ by $N=S \circ \mathcal{F}$ ( $\mathscr{F}$ and $S$ are given in (1.11) and (1.12) respectively). Suppose

$$
\left\{\begin{array}{l}
\text { there exists a nonempty, closed, convex set } X \text { of } C([0, T], E) \\
\text { such that } X \text { is mapped into itself by the multi } N \text { and also } \\
N(X) \text { is a subset of a bounded set in } C([0, T], E)
\end{array}\right.
$$

holds. Then (1.2) has a solution in $W^{1,1}([0, T], E)$.

Proof: The result follows from Theorem 2.1 in [6]. In [6] we assumed $X$ was bounded (here we assume $N(X)$ is a subset of a bounded set in $C([0, T, E)$ ); however the proof is the same.

Corollary 1.4: Let $E$ be a separable Banach space and let $F:[0, T] \times E \rightarrow C K(E)$ satisfy (1.4), (1.8) and (1.9). Then (1.2) has a solution in $W^{1,1}([0, T], E)$.

Proof: The result follows from Theorem 1.3. Notice (1.13) holds with $X=$ $C([0, T], E)$.

Theorem 1.5: Let $E$ be a separable Banach space and let $F:[0, T] \times E \rightarrow C(E)$ satisfy (1.7), (1.9) and either (1.5) or (1.6). Define the operator $N=S \circ \mathcal{F}$ (F and $S$ are given in (1.11) and (1.12) respectively) and suppose (1.13) holds. Then (1.2) has a solution in $W^{1,1}([0, T], E)$.

Corollary 1.6: Let $E$ be a separable Banach space and let $F:[0, T] \times E \rightarrow C(E)$ satisfy (1.8), (1.9) and either (1.5) or (1.6). Then (1.2) has a solution in $W^{1,1}([0, T], E)$. 


\section{Differential Inclusions on Proximate Retracts}

In this section we study the existence of viable solutions $x:[0, T] \rightarrow K \subseteq E$ to the differential inclusion

$$
\left\{\begin{array}{l}
x^{\prime}(t) \in \phi(t, x(t)) \quad \text { a.e. } t \in[0, T] \\
x(0)=y_{0} \in K
\end{array}\right.
$$

By a solution (viable) to $(2.1)$ we mean a $x \in W^{1,1}([0, T], E)$ with $x^{\prime} \in \phi(t, x)$ a.e. on $[0, T], x(0)=y_{0}$ and $x(t) \in K$ for $t \in[0, T]$. Throughout this section we assume

$K$ is a proximate retract.

Definition 2.1: [9] A nonempty closed subset $K$ of $E$ is said to be a proximate retract if there exists an open neighborhood $U$ of $K$ in $E$ and a continuous (singlevalued) mapping $r: U \rightarrow K$ (called a metric retraction) such that the following two conditions are satisfied:

(i) $\quad r(x)=x$ for all $x \in K$;

(ii) $\quad\|r(x)-x\|=\operatorname{dist}(x, K)$ for all $x \in U$.

Remark 2.1: Now since we can take a sufficiently small $U$ (for example by restricting $U$ to $U \cap\{y \in E$ : $\operatorname{dist}(y, K)<\delta\}$ for some given $\delta>0$ ) we may assume (and we do so) that $\|r(u)-u\| \leq \delta$ for all $u \in U$.

For most of this section we will assume $\phi$ satisfies either

$$
\left\{\begin{array}{c}
\phi:[0, T] \times K \rightarrow C K(E) \text { satisfies }(1.4) \text { and }(1.7) \\
(\text { here } F \text { is replaced by } \phi \text { and } E \text { is replaced by } K \text { ) }
\end{array}\right.
$$

or

$$
\left\{\begin{array}{c}
\phi:[0, T] \times K \rightarrow C(E) \text { satisfies }(1.7) \text { and either }(1.5) \text { or }(1.6) \\
\text { (here } F \text { is replaced by } \phi \text { and } E \text { is replaced by } K \text { ). }
\end{array}\right.
$$

Now let $U$ be a fixed neighborhood of $K$ (chosen as in Remark 2.1) and let $\lambda$ be an Urysohn function for $(K, E \backslash U)$ with $\underset{\sim}{\lambda}(x)=1$ if $x \in K$ and $\lambda(x)=0$ if $x \notin U$. Let $r$ : $U \rightarrow K$ be a metric retraction. Define $\widetilde{\phi}:[0, T] \times E \rightarrow C(E)$ by

$$
\tilde{\phi}(t, x)=\left\{\begin{array}{cc}
\lambda(x) \phi(t, r(x)) & \text { if } x \in U \\
\{0\} & \text { if } x \notin U .
\end{array}\right.
$$

Remark 2.2: If $\phi$ satisfies (2.3) then $\tilde{\phi}$ satisfies (1.4) and (1.7) (with $F$ replaced by $\widetilde{\phi})$. A similar remark applies if $\phi$ satisfies (2.4).

Assume also that

where

$$
\phi(t, x) \subseteq T_{K}(x) \text { for all } x \in K \text { and a.e. } t \in[0, T]
$$

$$
T_{K}(x)=\left\{v \in E: \liminf _{t \rightarrow 0^{+}} \frac{\operatorname{dist}(x+t v, K)}{t}=0\right\}
$$


is the Bouligand tangent cone to $K$ at $x$.

We now concentrate our study on the differential inclusion

$$
\left\{\begin{array}{l}
x^{\prime}(t) \in \tilde{\phi}(t, x(t)) \quad \text { a.e. } t \in[0, T] \\
x(0)=y_{0} \in K
\end{array}\right.
$$

Notice any solution of (2.6) is a viable solution of (2.1); to see this notice if $x$ is a solution of $(2.6)$ then $x(t) \in K$ for all $t \in[0, T]$ by Theorem 3.1 of [7] (note (2.5) holds) so $\tilde{\phi}(t, x(t))=\lambda(x(t)) \phi(t, r(x(t)))=\phi(t, x(t))$. Conversely if $y$ is a viable solution of (2.1) then $y$ is a solution of (2.6).

Theorem 2.1: Let $E=(E,\|\cdot\|)$ be a separable Banach space and let $\tilde{\phi}, U$ be as above (in particular $U$ is chosen as in Remark 2.1 ).

(i) Suppose (2.2), (2.3), (2.5) and

$$
\left\{\begin{array}{l}
\text { there exists } \gamma \geq 0 \text { with } 2 \gamma T<1 \text { and with } \\
\quad \alpha(\tilde{\phi}([0, t] \times \Omega)) \leq \gamma \alpha(\Omega) \text { for any bounded subset } \Omega \text { of } E
\end{array}\right.
$$

hold. In addition suppose there is a constant $M$, independent of $\mu$, with $\|y\|_{0} \neq M$ for any solution $y \in W^{1,1}([0, T], E)$ to

$$
\left\{\begin{array}{l}
x^{\prime}(t) \in \mu \tilde{\phi}(t, x(t)) \quad \text { a.e. } t \in[0, T] \\
x(0)=y_{0} \in K
\end{array}\right.
$$

for each $\mu \in(0,1)$. Thus $(2.1)$ has a viable solution $u$ with $\|u\|_{0} \leq M$.

(ii) Suppose (2.2), (2.4), (2.5) and (2.7) hold. In addition assume there is a constant $M$, independent of $\mu$, with $\|y\|_{0} \neq M$ for any solution $y \in W^{1,1}([0, T], E)$ to $(2.8)_{\mu}$ for each $\mu \in(0,1)$. Thus $(2.1)$ has a viable solution $u$ with $\|u\|_{0} \leq M$.

Proof: From Theorem 1.1 (if we are discussing $(i)$ ) or Theorem 1.2 (if we are discussing (ii)) we have immediately that $(2.8)_{1}$ has a solution $y$ (note $y(t) \in K$ for all $t \in[0, T]$ by Theorem 3.1 of [7]). Thus $y$ is a solution of (2.1).

Remark 2.3: Suppose $E$ is a Hilbert space and $K$ is a closed, convex subset of $E$. In addition, suppose $\phi$ satisfies (1.9) (with $F$ replaced by $\phi$ and $E$ replaced by $K$ ). Then (2.7) is satisfied. To see this notice $r$ in this case is nonexpansive. Now if $\Omega$ is a bounded subset of $E$ then since

we have

$$
\tilde{\phi}([0, t] \times \Omega) \subseteq \overline{c o}(\phi([0, t] \times r(\Omega)) \cup\{0\})
$$

$$
\alpha(\tilde{\phi}([0, t] \times \Omega)) \leq \alpha(\phi([0, t] \times r(\Omega))) \leq \gamma \alpha(r(\Omega)) \leq \gamma \alpha(\Omega)
$$

It is also possible to use Corollary 1.4 or Corollary 1.6 to establish an existence principle for (2.1). Suppose there is a constant $M$ with $\|y\|_{0}<M$ for any possible viable solution to $(2.1)$. Let $\epsilon>0$ be given and let $\tau_{\epsilon}: E \rightarrow[0,1]$ be the Urysohn function for

$$
(\bar{B}(0, M), E \backslash B(0, M+\epsilon))
$$


$\underset{\sim}{\text { such that }} \tau_{\epsilon}(x)=1$ if $\|x\| \leq M \quad$ and $\tau_{\epsilon}(x)=0 \quad$ if $\|x\| \geq M+\epsilon$. Let $\tilde{\phi}_{\epsilon}(t, x)=\tau_{\epsilon}(x) \tilde{\phi}(t, x)$ and we look at the differential inclusion

$$
\left\{\begin{array}{c}
x^{\prime}(t) \in \tilde{\phi}_{\epsilon}(t, x(t)) \quad \text { a.e. } t \in[0, T] \\
x(0)=y_{0} .
\end{array}\right.
$$

Theorem 2.2: Let $E=(E,\|\cdot\|)$ be a separable Banach space and assume (2.2) and (2.5) hold. In addition, suppose $\phi:[0, T] \times K \rightarrow C(E)$ satisfies either (2.3) or (2.4). Assume there is a constant $M$ with $\|y\|_{0}<M$ for any possible viable solution $y \in W^{1,1}([0, T], E)$ to (2.1). Let $\epsilon>0$ be given and let $\tau_{\epsilon}, \tilde{\phi}_{\epsilon}$ be as above. Suppose

$$
\left\{\begin{array}{l}
\text { there exists } \gamma \geq 0 \text { with } 2 \gamma T<1 \text { and with } \\
\alpha\left(\tilde{\phi}_{\epsilon}([0, t] \times \Omega)\right) \leq \gamma \alpha(\Omega) \text { for any bounded subset } \Omega \text { of } E
\end{array}\right.
$$

holds, and in addition, assume $\|w\|_{0}<M$ for any possible solution $w \in$ $W^{1,1}([0, T], E)$ to $(2.9)$. Then $(2.1)$ has a viable solution $u$ with $\|u\|_{0}<M$.

Proof: From Corollary 1.4 or Corollary 1.6 (note (1.8) is satisfied with $F$ replaced by $\left.\tilde{\phi}_{\epsilon}\right)$ we have immediately that $(2.9)$ has a solution $y$. By assumption $\|y\|_{0}<M$ and so by definition $\tilde{\phi}_{\epsilon}(t, y(t))=\tilde{\phi}(t, y(t))$. Thus $y$ is a solution of (2.6). Now Theorem 3.1 of [7] implies $y(t) \in K$ for every $t \in[0, T]$ and so $y$ is a solution of (2.1).

Finally, in this section we examine the differential inclusion

$$
\left\{\begin{array}{l}
x^{\prime} \in \overline{\exp } \overline{\operatorname{conv}} \phi(t, x) \quad \text { a.e. on }[0, T] \\
x(0)=y_{0} \in K
\end{array}\right.
$$

New results will be obtained for (2.11) (these extend and complement results in the literature [1-3] and these automatically lead to new existence criterion for (2.1).

For the remainder of this section we will let

$$
G(t, x)=\overline{\exp } \overline{\operatorname{conv}} \phi(t, x) .
$$

As before, $K$ will be a proximate retract (i.e. (2.2) holds). We also assume the following conditions hold:

$$
\begin{gathered}
\phi:[0, T] \times K \rightarrow C(E) \\
x \mapsto \overline{\operatorname{conv}} \phi(t, x) \text { is continuous for a.e. } t \in[0, T] \\
(t, x) \mapsto G(t, x)=\overline{\exp } \overline{\operatorname{conv}} \phi(t, x) \text { is } \mathcal{L} \otimes \mathfrak{B} \text { measurable }
\end{gathered}
$$

for a.e. $t \in[0, T]$ and every $x \in K$ with $\|x\| \leq r$

and

$$
G(t, x) \subseteq T_{K}(x) \text { for all } x \in K \text { and a.e. } t \in[0, T]
$$

$$
\overline{\phi(t, K)} \text { is compact for a.e. } t \in[0, T] \text {. }
$$


Recall the following results [3, pp. 71-72].

Theorem 2.3: (Krein-Milman) Let $X$ be a Banach space and $\emptyset \neq M \subseteq X$ be compact. Then $\overline{\exp } \overline{\operatorname{conv}} M$ is the smallest closed subset of $M$ such that

$$
\overline{\operatorname{conv}} \overline{\exp } \overline{\operatorname{conv}} M=\overline{\operatorname{conv}} M \text {. }
$$

Theorem 2.4: Let $X$ be a Banach space, $D \subseteq X$ be closed and suppose $F: D \rightarrow 2^{X}$ have closed values with $\overline{F(D)}$ compact and $\overline{\operatorname{conv}} F$ continuous. Then $\overline{\exp } \overline{\operatorname{conv}} F$ is l.s.c.

Remark 2.4: Notice (2.12) implies $G:[0, T] \times K \rightarrow C(E)$.

Remark 2.5: By Theorem 2.3 for a.e. $t \in[0, T]$ we have

$$
\overline{\exp } \overline{\operatorname{conv}} \phi(t, x) \subseteq \phi(t, x) .
$$

Thus if we assume (1.7) (with $F$ replaced by $\phi$ ) then (2.15) is automatically satisfied. In addition if we assume (2.5) then (2.16) holds.

Let $U, \lambda$ and $r$ be as in the beginning of Section 2 and define $\widetilde{G}:[0, T] \times E \rightarrow C(E)$ by

$$
\widetilde{G}(t, x)=\left\{\begin{array}{cc}
\lambda(x) G(t, r(x)) & \text { if } x \in U \\
\{0\} & \text { if } x \notin U
\end{array}\right.
$$

Theorem 2.5: Let $E=(E,\|\cdot\|)$ be a separable Banach space and let $G, \widetilde{G}, U$, $\lambda, r$ be as above. Assume (2.2), (2.12), (2.13), (2.14), (2.15), (2.16), (2.17) and

$$
\left\{\begin{array}{l}
\text { there exists } \gamma \geq 0 \text { with } 2 \gamma T<1 \text { and with } \\
\alpha(\tilde{G}([0, t] \times \Omega)) \leq \gamma \alpha(\Omega) \text { for any bounded subset } \Omega \text { of } E
\end{array}\right.
$$

hold. In addition, suppose there is a constant $M$, independent of $\mu$, with $\|y\|_{0} \neq M$ for any solution $y \in W^{1,1}([0, T], E)$ to

$$
\left\{\begin{array}{l}
x^{\prime}(t) \in \mu \widetilde{G}(t, x(t)) \quad \text { a.e. } t \in[0, T] \\
x(0)=y_{0} \in K
\end{array}\right.
$$

for each $\mu \in(0,1)$. Thus (2.11) (and also (2.1)) has a viable solution $u$ with $\|u\|_{0} \leq M$.

Proof: We will apply Theorem $2.1($ ii $)$. Let $N$ be a null set and suppose

and

$$
x \mapsto \overline{\operatorname{conv}} \phi(t, x) \text { is continuous for } t \in[0, T] \backslash N
$$

$$
\overline{\phi(t, K)} \text { is compact for } t \in[0, T] \backslash N \text {. }
$$

Fix $t \in[0, T] \backslash N$. Now $\phi(t, \cdot): K \rightarrow C(E)$ with $\overline{\operatorname{conv}} \phi(t, \cdot)$ continuous and $\overline{\phi(t, K)}$ compact. Theorem 2.4 implies that $\overline{\exp } \overline{\operatorname{conv}} \phi(t, \cdot)$ is l.s.c. for $t \in[0, T] \backslash N$. Consequently

$$
x \mapsto G(t, x) \text { is l.s.c. for a.e. } t \in[0, T] \text {. }
$$


Now (2.14), (2.15), (2.16), (2.18), (2.20) and Theorem 2.1 (ii) imply that (2.11) has a viable solution $u$ with $\|u\|_{0} \leq M$ (in addition for fixed $t \in[0, T] \backslash N$ we have from Theorem 2.3 that $\overline{\exp } \overline{\operatorname{conv}} \phi(t, u(t)) \subseteq \phi(t, u(t)$ ) (note $\phi(t, u(t)$ ) is nonempty and compact valued) and so $u$ is a solution of (2.1)).

Remark 2.6: Notice (2.13) and (2.17) could be removed if we assume (2.20).

Suppose there is a constant $M$ with $\|y\|_{0}<M$ for any possible viable solution to (2.11). Let $\epsilon>0$ be given and let $\tau_{\epsilon}: E \rightarrow[0,1]$ be as before. Let $\widetilde{G}_{\epsilon}(t, x)=$ $\tau_{\epsilon}(x) \widetilde{G}(t, x)$ and we now look at the differential inclusion

$$
\left\{\begin{array}{c}
x^{\prime}(t) \in \widetilde{G}_{\epsilon}(t, x(t)) \quad \text { a.e. } t \in[0, T] \\
x(0)=y_{0}
\end{array}\right.
$$

Now Theorem 2.2 (together with the ideas in Theorem 2.5) immediately yields the following result.

Theorem 2.6: Let $E=(E,\|\cdot\|)$ be a separable Banach space and assume (2.2), (2.12)-(2.17) hold. Assume there is a constant $M$ with $\|y\|_{0}<M$ for any possible viable solution $y \in W^{1,1}([0, T], E)$ to $(2.11)$. Let $\epsilon>0$ be given and let $\tau_{\epsilon}, \widetilde{G}_{\epsilon}$ be as above. Suppose

$$
\left\{\begin{array}{l}
\text { there exists } \gamma \geq 0 \text { with } 2 \gamma T<1 \text { and with } \\
\alpha\left(\tilde{G}_{\epsilon}([0, t] \times \Omega)\right) \leq \gamma \alpha(\Omega) \text { for any bounded subset } \Omega \text { of } E
\end{array}\right.
$$

holds, and in addition assume $\|w\|_{0}<M$ for any possible solution $w \in$ $W^{1,1}([0, T], E)$ to (2.21). Then (2.11) (and also (2.1)) has a viable solution $u$ with $\|u\|_{0}<M$.

Finally it is worth noting that it is possible to obtain an existence principle of the type in Theorem 1.2 for the differential inclusion

Let

$$
\left\{\begin{array}{l}
x^{\prime} \in \overline{\exp } \overline{\operatorname{conv}} F(t, x) \quad \text { a.e. on }[0, T] \\
x(0)=x_{0} \in E
\end{array}\right.
$$

$$
H(t, x)=\overline{\exp } \overline{\operatorname{conv}} F(t, x)
$$

and assume the following conditions are satisfied:

$$
F:[0, T] \times E \rightarrow C(E)
$$

$x \mapsto \overline{\operatorname{conv}} F(t, x)$ is continuous for a.e. $t \in[0, T]$

$$
(t, x) \mapsto H(t, x)=\overline{\exp } \overline{\operatorname{conv}} F(t, x) \text { is } \mathcal{L} \otimes \mathscr{B} \text { measurable }
$$

$\left\{\begin{array}{l}\text { for each } r>0 \text { there exists } h_{r} \in L^{1}[0, T] \text { such that }\|H(t, x)\| \leq h_{r}(t) \\ \text { for a.e. } t \in[0, T] \text { and every } x \in E \text { with }\|x\| \leq r\end{array}\right.$

and $\quad\left\{\begin{array}{l}\text { there exists } \gamma \geq 0 \text { with } 2 \gamma T<1 \text { and with } \\ \alpha(H([0, t] \times \Omega)) \leq \gamma \alpha(\Omega) \text { for any bounded subset } \Omega \text { of } E\end{array}\right.$ 


$$
\overline{F(t, E)} \text { is compact for a.e. } t \in[0, T] \text {. }
$$

Theorem 2.7: Let $E=(E,\|\cdot\|)$ be a separable Banach space and assume (2.24)(2.29) hold. In addition, suppose there is a constant $M_{0}$, independent of $\lambda$, with $\|y\|_{0} \neq M_{0}$ for any solution $y \in W^{1,1}([0, T], E)$ to

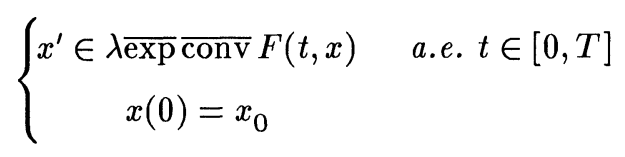

for each $\lambda \in(0,1)$. Thus (2.23) (and so (1.2)) has a solution $u \in W^{1,1}([0, T], E)$ with $\|u\|_{0} \leq M$.

Proof: As in Theorem 2.5 it is easy to see that

$$
x \mapsto H(t, x) \text { is l.s.c. for a.e. } t \in[0, T] \text {. }
$$

Now apply Theorem 1.2 to $H$.

Remark 2.7: We could also obtain an analog of Theorem 1.5 and Corollary 1.6 for the differential inclusion (2.23).

Remark 2.8: Notice (2.25) and (2.29) could be removed if we assume (2.31).

\section{References}

[1] De Blasi, F.S. and Pianigiani, G., On the solution sets of nonconvex differential inclusions, J. Diff. Eqns. 128 (1996), 541-555.

[2] Deimling, K., Existence theorems for multivalued differential equations, Proc. Int. Symp. Nonlinear Analysis and Appl. to Biomathematics (ed. by J. Gopalakrishna and K. Murthy), Andhra Univ. Press, Visakhapatnam (1987), 35-52.

[3] Deimling, K., Multivalued Differential Equations, Walter de Gruyter, Berlin 1992.

[4] Frigon, M., Théoremes d'existence de solutions d'inclusions différentielles, Topological Methods in Diff. Eqns. and Inclusions (ed. by A. Granas and M. Frigon), NATO ASI Series C V 472, Kluwer Acad. Publ., Dordrecht (1995), 51-87.

[5] Frigon, M., Gorniewicz, L. and Kaczynski, T., Differential inclusions and implicit equations on closed subsets of $\mathbf{R}^{n}$, Proc. of the First World Congress of Nonlinear Analysts 1992 (ed. by V. Lakshmikantham) Vol. I-IV, Walter de Gruyter, Berlin (1996), 1797-1806.

[6] O'Regan, D., Multivalued integral equations in finite and infinite dimensions, Comm. in Appl. Analysis 2 (1998), 487-496.

[7] O'Regan, D., Viable solutions of differential equations and inclusions on proximate retracts in Banach spaces, Pan American Math J. (to appear).

[8] O'Regan, D., Nonlinear alternatives for multivalued maps with applications to operator inclusions in abstract spaces, Proc. Amer. Math. Soc., to appear.

[9] Plaskacz, S., On the solution sets of differential inclusions, Bollettino U.M.I. 6A (1992), 387-394. 


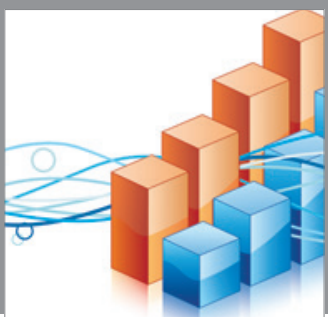

Advances in

Operations Research

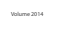

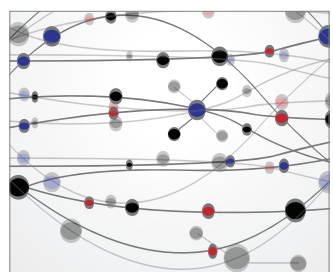

\section{The Scientific} World Journal
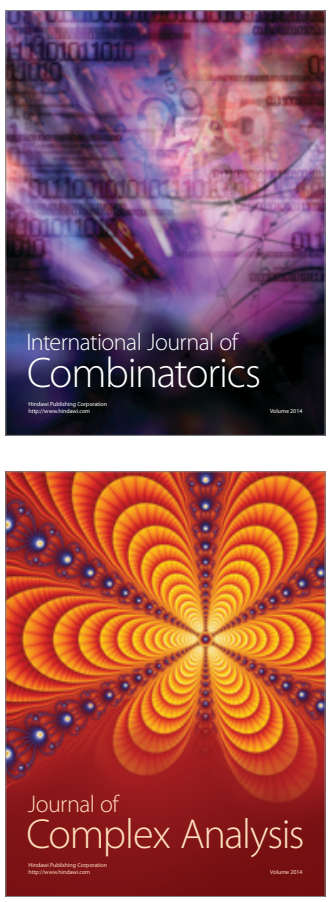

International Journal of

Mathematics and

Mathematical

Sciences
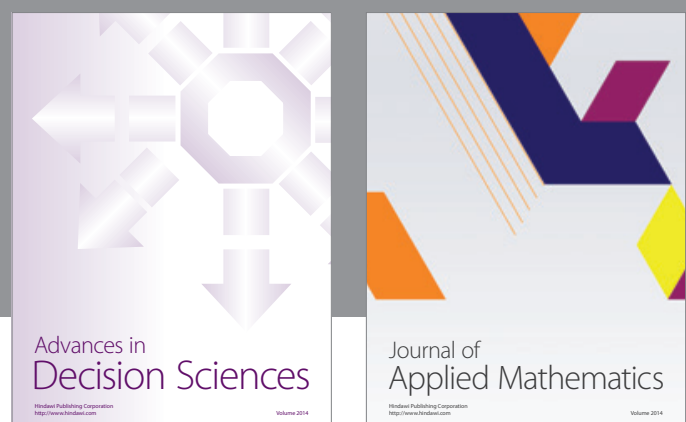

Journal of

Applied Mathematics
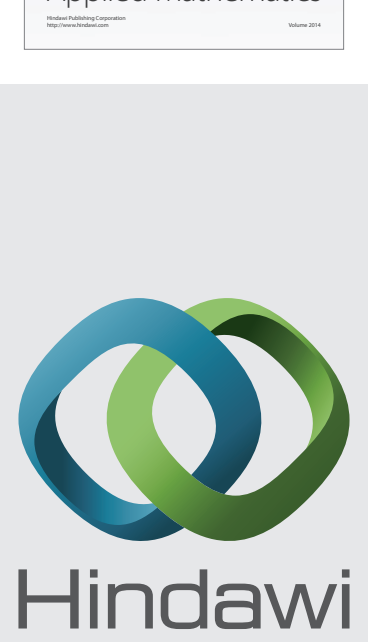

Submit your manuscripts at http://www.hindawi.com
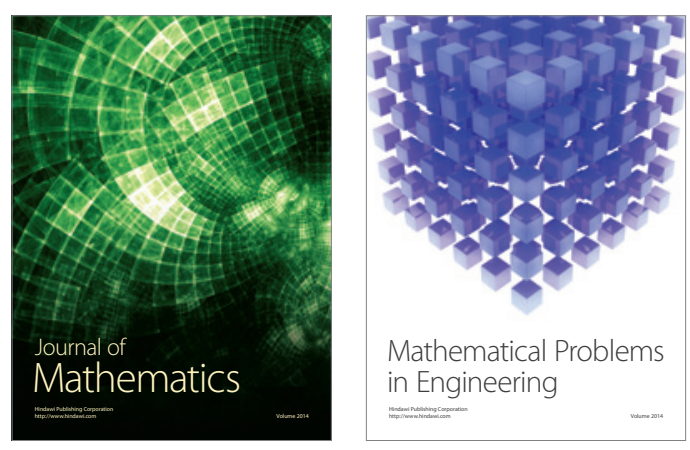

Mathematical Problems in Engineering
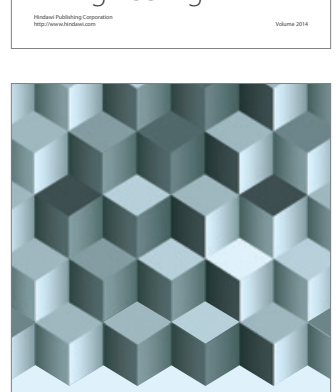

Journal of

Function Spaces
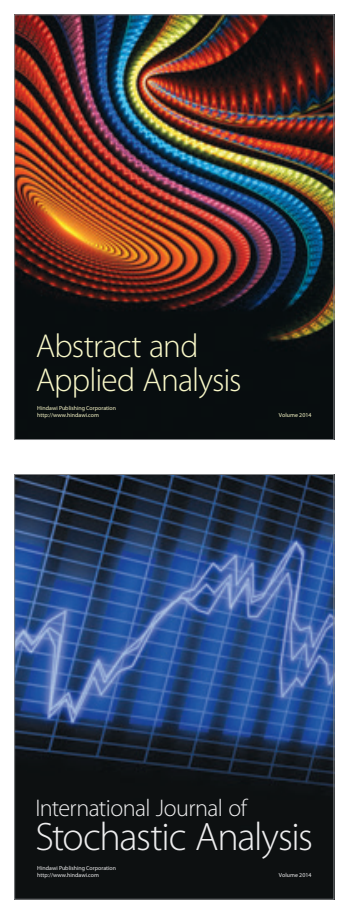

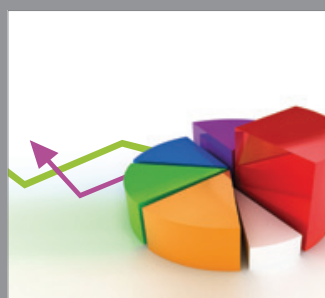

ournal of

Probability and Statistics

Promensencen
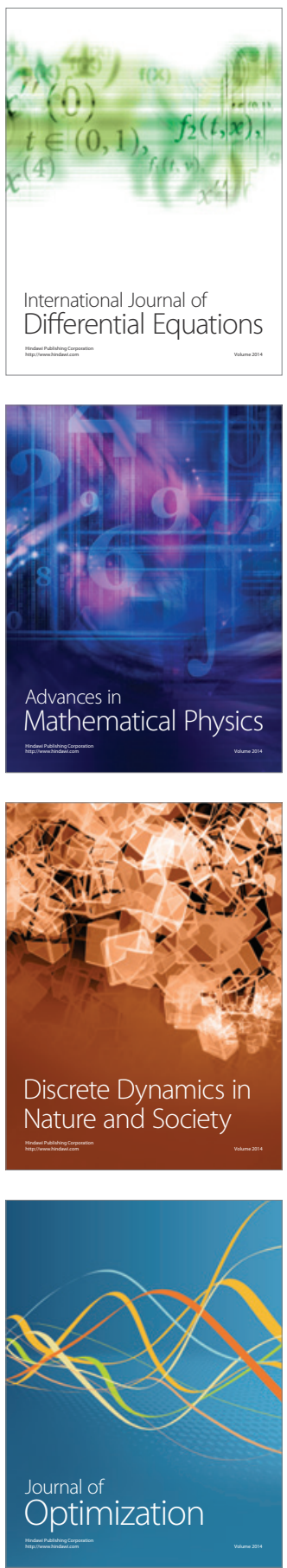